connectable by a straight line. Algebraically speaking, this requirement means that the following formula must be valid for all values of $x$ and $y$, when $u$ is determined by $x$ and $y$ according to the function $u=u(x, y)$ :

$$
\frac{h_{u}(u(x, y))-h_{x}(x)}{t}=\frac{h_{y}(y)-h_{x}(x)}{T}
$$

In our case, $u(x, y)$ equals $x / y$. If this function and the formulas [13] are inserted into the basic equation [14], we obtain:

$$
\begin{gathered}
\frac{p_{u} \cdot \log x-p_{u} \cdot \log y+q_{u}-p_{x} \cdot \log x-q_{x}}{t}= \\
\frac{p_{y} \cdot \log y+q_{y}-p_{x} \cdot \log x-q_{x}}{T}
\end{gathered}
$$

since $\log x / y=\log x-\log y$.

The equation [15] must be valid for all values of $x$ and $y$. Hence, the coefficients of $\log x$ and $\log y$ and the terms not containing $x$ and $y$ on both sides of [15] must be equal. For the eight parameters $p_{x}, p_{y}, p_{u}, q_{x}, q_{y}, q_{u}, t$ and $T$, we obtain:

$$
\begin{aligned}
& \mathrm{T} \cdot\left(\mathrm{p}_{\mathrm{u}}-\mathrm{p}_{\mathrm{x}}\right)=-\mathrm{t} \cdot \mathrm{p}_{\mathrm{x}} \\
& \mathrm{T} \cdot \mathrm{p}_{\mathrm{u}}=\mathrm{t} \cdot \mathrm{p}_{\mathrm{y}} \\
& \mathrm{T} \cdot\left(\mathrm{q}_{\mathrm{u}}-\mathrm{q}_{\mathrm{x}}\right)=\mathrm{t} \cdot\left(\mathrm{q}_{\mathrm{y}}-\mathrm{q}_{\mathrm{x}}\right) .
\end{aligned}
$$

$\mathrm{I}_{\mathrm{n}}$ addition to [16], certain requirements as to length and range of the scales may be specified. However, such requirements must not conflict with [16].

To fix the end points of the scales, we must return to the original meaning of $x$ and $y, x$ representing the optical density of the sample at 600 and $y$ at $505 \mathrm{~nm}$. For the applications discussed here, $\mathrm{x}$ and $\mathrm{y}$ may vary within the limits

$$
0.1 \leqq \mathrm{x} \leqq 0.6 \text { and } 0.8 \leqq \mathrm{y} \leqq 1.1 \text {. }
$$

If, for instance, we demand $h_{x}(0.1)=0, p_{x}$ and $q_{x}$ must satisfy the condition:

and, since $\log 0.1=-1$,

$$
0=\mathrm{p}_{\mathbf{x}} \cdot \log 0.1+\mathrm{q}_{\mathrm{x}}
$$

$$
\mathrm{p}_{\mathrm{x}}=\mathrm{q}_{\mathrm{x}}
$$

Depending on the required specifications for length and range of the scales, different values for the parameters are obtained. $A^{\text {. }}$ useful solution is obtained if, in addition to $h_{x}(0.1)=0$, we require $h_{y}(1.1)=0$ and utilize simple values for the parameters. Without entering further into the derivation, we give the solution for the scale measures:

$$
\begin{aligned}
& h_{x}(s)=2 \log 10 s \\
& h_{y}(s)=10 \log \frac{1.1}{s} \\
& h_{u}(s)=\frac{10}{6} \log 11 s \\
& T: t=6: 1 .
\end{aligned}
$$

Selecting $15 \mathrm{~cm}$ as a unit, we can easily encompass all three scales into a page size DIN A 4. For the scale values, we obtain the following lengths in $\mathrm{cm}$ :

$$
\begin{aligned}
& h_{x}(s)=30 \log 10 s \\
& h_{y}(s)=150 \log \frac{1.1}{s} \\
& h_{u}(s) \doteq 25 \log 11 s .
\end{aligned}
$$

The scale distance chosen was $T=15 \mathrm{~cm}, t=2.5 \mathrm{~cm}$.

As an example of the calibration of the scales according to [18], we have: On the $x$-scale (representing $D_{R}$ ), the following distance $\mathrm{h}_{\mathrm{x}}$ in $\mathrm{cm}$ above the baseline is obtained for $\mathrm{x}=0.5$ :

$$
\mathrm{h}_{\mathrm{x}}(0.5)=30 \cdot \log 5=20.97 \mathrm{~cm} \text {. }
$$

The calculations of the scale values were performed on a computer in steps of 0.01 , beginning at 0.1 and ending at 0.6 for the $x$-scale $\left(D_{R}\right)$; and beginning at 0.8 and ending at 1.1 for the $y$-scale $\left(D_{G}\right)$. An additional feature of the $y$-scale is that on this scale, the values for $D_{G}$ decrease from below upwards.

This work was supported by the „Schweizerischer Nationalfond zur Förderung der wissenschaftlichen Forschung“, Proj. Nr. 4517.

\section{References}

1. Lundsganad-Hansen, P. and U. Schreiber, diese Z. 6,197 (1968).

Dipl.-Math. H. Ehrengruber Inselspital

CH 3008 Berne (Switzerland)

\title{
Photometrische Mikrobestimmung von Kohlenmonoxid in Luft und Blut
}

\author{
Von G. Ciuhandu und V. Rusu
}

Aus dem Laboratorium für Toxikologie des Institutes für Hygiene (Direktor: Dr. E. Andriescu), Timișoara, Rumänien

(Eingegangen am 18. April 1967)

Die Farbreaktion der alkalischen Lösung von Na-Silbersulfamidbenzoat mit CO gestattet die Bestimmung von CO-Spuren in Luft bzw. im Blut mit einem einfachen Verfahren.

Durch 20stdg. Kontakt von $2 \mathrm{~m} l$ Reagenz mit $10 \mathrm{~m} l$ Luft und nachfolgendes Photometrieren des Reaktionsgemisches bei $420 \mathrm{~nm}$ kann CO in Luft in Konzentrationen von 20-2000 ppm bestimmt werden.

Nach Frcisetzen aus $0,1-0,2 \mathrm{~m} /$ Blut mit Kaliumferricyanid und Saponin und 20stdg. Diffusion in $2 \mathrm{~m} l$ Reagenz kann CO im Blut bis zu 0,2 Vol-\% bestimint werden.

Die Diffusionsdauer kann durch mechanisches Schütteln erheblich abgekürzt werden. Die Reaktion wird nach 5 Stdn. quantitativ und die Steigung der Eichkurve, somit auch die Empfindlichkeit der Bestimmung, wird um etwa $60 \%$ erhöht.

Die Methoden eignen sich gut für Serienbestimmungen.

Traces of $\mathrm{CO}$ in the atmosphere or in blood can be determined by a simplified procedure based on the colour reaction of an alkaline solution of Na-silver-sulphamide-benzoate with $\mathrm{CO}$.

Reagent $(2 \mathrm{ml})$ and air $(10 \mathrm{ml})$ are left in contact for $20 \mathrm{hr}$. The extinction of the solution is then measured at $420 \mathrm{~nm}$. In this way $20-2000$ ppm $\mathrm{CO}$ in the air can be determined.

$\mathrm{CO}$ can be measured in blood down to a concentration of $0.2 \mathrm{Vol}-\% ; 0.1-0.2 \mathrm{ml}$. of blood are treated with potassium ferricyanide and saponin to release the $C O$, which is then diffused over $20 \mathrm{hr}$. into $2 \mathrm{ml}$ of reagent.

The time of diffusion can be considerably reduced by shaking: the reaction is complete after $\mathbf{5}$ hr. and the slope of the calibration curve, and thus the sensitivity, is increased by $60 \%$. The methods are suitable for serial assays. 
Die Diagnose von CO-Vergiftungen wird im allgemeinen durch Bestimmung des Gases im Blut und seltener retrospektiv durch Analyse von atmosphärischen Luftproben aufgestellt.

Die Bestimmung des Gases in der Luft durch Oxydation mit Hopkalit oder Hoolamit ${ }^{1}$ ) bzw. durch die IR-Absorptionsanalyse stellen klassische Methoden dar, die aber eine Apparatur spezieller Konstruktion erfordern, welche oft schwer zugänglich ist. Ein anderes Verfahten beruht auf der Reaktion zwischen $\mathrm{PdCl}_{2}$ und $\mathrm{CO}$ mit Berechnung des reduzierten Metalls durch Differenzbestimmung (1). Diese indirekte Bestimmung kann vermieden werden, wenn man zum Pd-Salz Phosphomolybdänsäure hinzufügt, welche zu Molybdänblau reduziert wird (2). SHEPHERD und Mitarbeiter verwenden.Indikatorröhrchen mit einer Fülluing von Silikagel, Silikomolybdat und Pd-Salz-Katalysator $(3,4)$.

Für die Blutanalyse benutzte Methoden beruhen auf der Austreibung der Gase und Messung des CO-Volumens nach Absorption der anderen Komponenten (5) oder der Ablesung der eudiometrischen Volumenkontraktion (6) bzw. der CO-Absorption in geeigneten Reagentien (7). In den Originalvorschriften verwenden die Autoren verhältnismäßig erhebliche Blutmengen, um eine zufriedenstellende Genauigkeit zu erreichen. ChristmanN und RANDALL fangen die extrahierten Gase in einem Absorptionsgefäß, das eine bekannte Menge $\mathrm{PdCl}_{2}$ enthält, auf, bestimmen das reduzierte Pd und aus diesem die CO-Menge (8). Die Empfindlichkeit steigt bei der Auswertung des extrahierten $\mathrm{CO}$ mit Hilfe eines IR-Analysators $(9,10)$. Andere Verfahren, bei denen das Gas nach Freisetzung aus dem Blut mit reiner Luft über ein mit $\mathrm{PdCl}_{2}$ getränktes Filterpapier $(11,12)$ oder durch ein Indikator-Röhrchen $\left.{ }^{2}\right)$ geleitet wird (13), scheinen nur orientierenden Wert zu besitzen $(14,15)$. Dagegen erwies sich das mikrogasvolumetrische Verfahren von Roughton und Scholander (16) als sehr empfindlich und bei genügender manueller Geschicklichkeit als sehr genau (14).

Andere Bestimmungsmethoden verwerten die spezifischen physikalischen oder chemischen Eigenschaften von $\mathrm{HbCO}$. Hier sind vor allem die optischen Methoden zu erwähnen, die sich auf die maximale Lichtabsorption von $\mathrm{HbCO}$ und $\mathrm{HbO}_{2}$ bzw. $\mathrm{Hb}$ bei verschiedenen Wellenlängen gründen. Sind in einer hämolysierten Blutlösung nur zwei Komponenten vorhanden, so kann $\mathrm{HbCO}$ spektrophotometrisch aufgrund einer einfachen Relation durch zwei Extinktionsmessungen ermittelt werden. Störende Ionen sind durch Maskieren zu entfernen (17-22). Nach WolfF (23) sind auch die iso-ionischen $\mathrm{pH}$-Werte des Ausflockens von $\mathrm{HbO}_{2}$ und $\mathrm{HbCO}$ verschieden. Nach Hämolyse, $5 \mathrm{Min}$. langem Erwärmen auf $55^{\circ}$ bei pH 5,1 und Filtrieren kann $\mathrm{HbCO}$ in der Lösung photometrisch ermittelt werden. Nach eingehenden Untersuchungen wies GrAMER darauf hin, daß annehmbare Ergebnisse nur durch strenges Einhalten der Arbeitsbedingungen erzielt werden können (14). Ein anderes Vorgehen stellt die direkte Diffusion von $\mathrm{CO}$ aus Blut im geschlossenen Raum in ein geeignetes Reagens dar. Das Gas wird mittels Ferricyanid-Saponin-Gemisch oder Ansäuren mit verd. Schwefelsäure freigesetzt. Als Reagens benutzt man $\mathrm{PdCl}_{2}$. $\mathrm{CO}$ wird aus der Menge des reduzierten Metalls oder aus der freigesetzten Säuremenge indirekt berechnet. Das. Verfahren wurde zum erstenmal von WENNESLAND vor über 25 Jahren angewendet (24) und von zahlreichen Autoren modifiziert (25-35). Die letzten Anteile von $C O$ diffundieren aber langsam aus dem Blut und sind nur schwer, selbst bei hohem Vakuum, zu entfernen $(9,10,14)$. Die Diffusion geringer CO-Mengen konnte noch 24 Stdn. nach der Freisetzung des Gases mit Ferricyanid-Saponin oder $\mathrm{H}_{2} \mathrm{SO}_{4}$ nachgewiesen wierden (36). Diese Tatsache macht die aus dem redu-

1) Hopkalit: Verschieden zusammengesetzte Gemische aus Kupferoxid und Mangandioxid (evtl. auch Kobaltoxiden und Silberoxid) zux Oxydation von $\mathrm{CO}$ zu $\mathrm{CO}_{2}$.

Hoolamit: $\mathrm{J}_{2} \mathrm{O}_{5}$-Suspension in rauchender Schwefelsäure, welche bei Zimmertemperatur $\mathrm{CO} \mathrm{zu} \mathrm{CO}_{2}$ oxidiert und. sich grün bzw. braun verfärbt.

2) Hersteller: Drägerwerk, Lübeck. zierten Pd stöchiometrisch berechnete CO-Menge zweifelhaft. Der Fehler kann nur durch vorhergehende Eichung mit Blutproben bekannten CO-Gehaltes vermieden werden. JoNES und WALTERS (37) wiesen aber darauf hin, daß solche Proben kaum aus 0 bzw. 100proz. gesättigten Blutproben herstellbar sind. Die Eichung geschieht am besten, indem man die Blutproben parallel mit einer objektiven Methode ( $v$. SLYKE (5)) analysiert (36). In einer verbesserten Modifikation eichen VIGNOLI und Mitarbeiter im Vergleich zur eudiometrischen Bestimmung (NicLoux (6)) der aus dem Blut extrahierten Gase (38). Statt das reduzierte Pd durch Differenz auszuwerten, lösen sie das Metall direkt in Phosphomolybdowolframsäure und photometrieren das gebildete Molybdänblau.

\section{Reagenz und Methodik}

In einer Reihe von Untersuchungen benutzten wir während der letzten 12 Jahre zum Nachweis und zur Bestimmung von $\mathrm{CO}$ die alkalische Lösung von $\mathrm{Na}$-Silbersulfamidbenzoat $(\mathrm{SSB})^{3}$ ) als neues Farbreagens. Dies erhäls man direkt aus $\mathrm{AgNO}_{3}, \mathrm{Na}$-p-Sulfamidbenzoat und $\mathrm{NaOH}$ nach folgender Gleichung

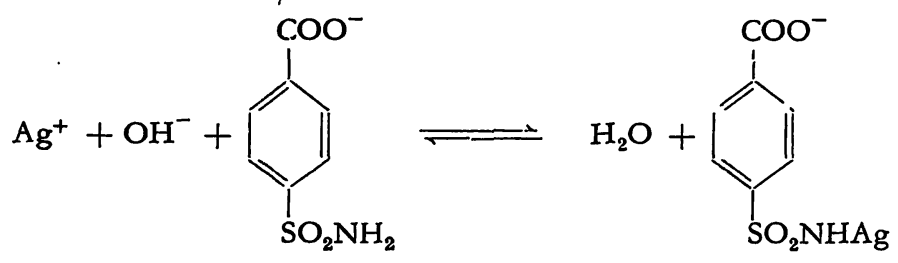

Das Gleichgewicht ist stark nach rechts verschoben. Die $\mathrm{Ag}^{+-}$Konzentration ist zu gering um $\mathrm{Ag}_{2} \mathrm{O}$ auszufällen, aber die Abhängigkeit zwischen $\mathrm{Ag}^{+}=$und $\mathrm{OH}^{-}$-Ionen kann durch Fällungsteaktion mit Halogeniden (39) oder potentiometrisch (40) verfolgt werden.

Bei Kontakt mit CO schlägt das farblose SSB sofort nach Gelb oder Braun um. Es entsteht ein durchsichtiges und sehr beständiges Silbersol, welches eine maximale Absorption bei $420 \mathrm{~nm}$ aufweist. Die Reaktion ist als Reduktion der Silberkationen zu formulieren:

$$
2 \mathrm{Ag}^{+}+\mathrm{CO}+4 \mathrm{OH}^{-} \longrightarrow 2 \mathrm{Ag}+\mathrm{CO}_{3}^{2-}+2 \mathrm{H}_{2} \mathrm{O}
$$

Durch Elektrophorese wurde die negative Ladung des Sol-Partikels nachgewiesen (41). Andererseits erwies sich die Beständigkeit des Sols gegen einen CO-Úberschuß wegen Entladung der am Partikel absorbierten $\mathrm{Ag}^{+-}$ Ionen als gering. Die Stabilität kann durch Adsorption von $\mathrm{Cu}^{2+}$-Ionen wieder hergestellt werden (42). Demzufolge wurde die Struktur des Partikels folgendermaßen dargestellt:

$$
\begin{gathered}
{\left[\mathrm{m}(\mathrm{Ag}) \mathrm{nAg}^{+} \cdot \mathrm{pOH}^{-} \cdot \mathrm{rH}_{2} \mathrm{~N} \cdot \mathrm{SO}_{2} \cdot \mathrm{C}_{6} \mathrm{H}_{4} \cdot \mathrm{COO}^{-}\right]-\mathrm{x}} \\
\mathrm{n}<\mathrm{p}+\mathrm{r} .
\end{gathered}
$$

In mit Carbonat alkalisierten Lösungen erhält man weniger beständige, purpurrote Sole. Durch nachfolgende Behandlung mit $\mathrm{NaOH}$ gehen diese in die oben erwähnten beständigen braunen Sole über. Dieses Verhalten spricht für eine Beteiligung der $\mathrm{OH}^{-}$-Ionen am Aufbau der Partikel des braunen Sols (43).

Die Reaktion zwischen SSB und CO verläuft infolge Verteilung des $\mathrm{CO} z$ wischen der gasförmigen und der flüssigen Phase gemäß dem Henry schen Gesetz. Dem-

3) Abkürzung: SSB = Natrium-Silber-Sulfamidbenzoat. 
entsprechend hängt die abgeschiedene Silbermenge nur von dem CO-Partialdruck $a b$ und bleibt unabhängig vom Luftdruck im Gasgemisch (44).

Die Empfindlichkeit der Reaktion erlaubt den Nachweis von 3 ppm CO in einem Liter Luft nach 20 stdg. Kontakt mit dem Reagens. Die Reaktion wird weder von Sauerstoff noch von zahlreichen anderen Gasen oder Dämpfen beeinflußt (39). Wasserstoff reduziert SSB etwa 700 mal langsamer bei Normaltemperatur, verliert aber diese Eigenschaft bei Eiskühlung. Leitet man Wasserstoff durch das eisgekühlte Reagenz, können COSpuren bis $5 \mathrm{ppm}$ nachgewiesen und bis zu $10 \mathrm{ppm}$ bestimmt werden (45). Das Durchperlen des Gases durch SSB-Lösung ist mit erheblichen Vorzügen gegenüber der klassischen Methode mit Hämoglobin zur Luftanalyse anwendbar, da der Farbumschlag nach Gelb visuell leicht zu erfassen ist (43). Durch 20stdg. Kontakt mit dem Reagenz unter leicht zu erfüllenden Versuchsbedingungen und Photometrieren des gebildeten Sols bei $420 \mathrm{~nm}$ kann CO in Luftproben bis $\mathrm{zu} 100 \mathrm{ml}$ (47) sogar in Gegenwart von Wasserstoff (48) aber auch in $\mathrm{CO}_{2}$ (44) und in $\mathrm{O}_{2}$ (49) bestimmt werden. Acetylen wird durch Leiten der Gase über mit Hg-Salzen imprägniertes Silikagel entfernt, während die Reaktionsdauer durch mechanisches Schütteln auf etwa 2 Stdn. verringert werden kann (50). Schwefelwasserstoff wird vorher in $\mathrm{KOH}$ zurückgehalten (43). Bei größeren CO-Konzentrationen kann die Messung auch im Bereich von $610 \mathrm{~nm}$ erfolgen (47).

In einer anderen Reihe von Arbeiten wurde SSB zur CO-Bestimmung in Blut, unter Diffusion des mit Ferricyanid und Saponin freigesetzten Gases, angewandt. Da die Gasdiffusion nicht quantitativ verläuft, führt man die Bestimmung anhand einer Eichkurve, die mit Blutproben bekannten CO-Inhaltes aufgestellt wurde (v. SLYKE), aus. Das Verfahren erwies sich als sehr empfindlich. Mit Diffusionssystemen von $50-100 \mathrm{~m} l$ (WIDMark oder WenNEsLand) und Messen nach 20 Stdn., kann $\mathrm{CO}$ bis unter $0,2 \mathrm{Vol}-\%$ in $0,5-1 \mathrm{ml}$ Blut gut bestimmt werden (36). In kleineren, $24 \mathrm{ml}$ fassenden Systemen, bestehend aus Paaren von Penicillingläschen, konnte bei einer Standardabweichung von 0,05 die Blutmenge auf $0,2 \mathrm{~m} /$ herabgesetzt werden (51). Unabhängig davon empfiehlt FELDSTEIN die Extraktion des Gases aus dem Blut und seine Bestimmung in einem Gemisch mit Luft unter Schütteln mit SSB (52).

In der vorliegenden Arbeit versuchten wir die Gasbestimmung für kleinste Luft- bzw. Blutmengen zu adaptieren. Für Blut wurde die Reaktion durch mechanisches Schütteln der Diffusionssysteme mit Blut und SSB beschleunigt.

\section{Durchführung der Bestimmung}

Die verwendeten Vorrichtungen sind aus Abbildung 1a (Luft) bzw. 1 b (Blut) in halbnatürlicher Größe ersichtlich. Sie wurden aus $12 \mathrm{~m} l$ fassenden Penicillingläsem gleicher Form und gleichen Inhalts hergestellt.

An das längere Röhrchen des Behälters a wird ein Gummischlauch angeschlossen und durch diesen die zu analysierende $L$ uft $4-5 \mathrm{mal}$ durchgespült. Enthält die Atmosphäre auch $\mathrm{H}_{2} \mathrm{~S}$, so wird die Luft

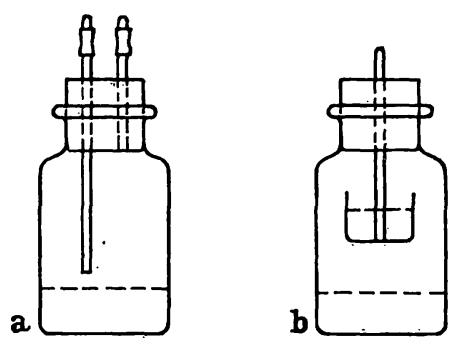

Abb. 1

Vorrichtungen zur CO-Bestimmung in Luft (a) bzw. im Blut (b)

durch eine vorgeschaltete kleine Waschflasche mit 20proz. $\mathrm{KOH}$ geleitet. Danach wird der Behälter sofort an beiden Öfnungen mit kleinen Kappen verschlossen.

Im Laboratorium führt man durch das längere Röhrchen $2 \mathrm{~m} l$ frische SSB-Lösung ein, verschließt sofort und läßt 20 Stdn. stehen. Als Leerwert verwendet man ein Volumen SSB in einem vollgefüllten Reagenzglas mit eingeschliffenem Stopfen. Am nächsten Tag photometriert man die Proben bei $420 \mathrm{~nm}$.

Zur Bestimmung des CO-Gehaltes im Blut benutzt man den Mikrodiffusor b. Ins Gläschen werden $2 \mathrm{~m} l \mathrm{SSB}$ eingeführt. In das aufgehängte Schiffchen mißt man $0,1 \mathrm{~m} / 5$ proz. Kaliumferricyanidund $0,1 \mathrm{~m} l 5$ proz. Saponinlösung, danach genau 0,1 bzw. $0,2 \mathrm{ml}$ Blut mit einer ausgezogenen Pipette ein, verschließt und mischt durch Umschwenken. Nach 20 Stdn. wird gegen eine Blindprobe ohne Blut photometriert.

In einer anderen Versuchsreihe wurden die Blutproben während einiger Stunden auf einer Schüttelmaschine horizontal geschüttelt. Es wurde die Abhängigkeit zwischen Lichtabsorption und Schütteldauer bzw. Gaskonzentration im Blut verfolgt.

Das Reagenz wurde immer frisch durch Schütteln von gleichen Volumina $0,1 \mathrm{M}$ Na-p-Sulfamidbenzoat- ${ }^{1}$ ) und $0,1 \mathrm{M}$ Silbernitratlösung in einem Glaszylinder mit eingeschliffenem Stopfen und Auflösen des gebildeten Niederschlags mit 0,5 Volumen $1 \mathrm{~N}$ Natronlauge, bereitet.

Die zur Aufstellung der Eichkurve nötigen CO-Luft- bzw. COBlut-Gemische werden in der früher beschriebenen Weise hergestellt $(49,51)$. Die Lichtabsorption wurde in Mikroküvetten mit einem Pulfrichphotometer $\left(S_{42}\right)$ gemessen.

Zur statistischen Auswertung werden Mittelwert $(\overline{\mathrm{x}})$, Streuung $\left(s=\sqrt{\frac{\Sigma\left(\bar{x}-x_{i}\right)^{2}}{n-1}}\right)$ und relative Streuung $\left(\frac{100 \cdot s}{\overline{\bar{x}}}\right)$ anhand einer Anzahl ( $\mathrm{n}$ ) von Bestimmungen berechnet.

\section{Ergebnisse}

In Gegenwart von CO schlägt die Farbe des Reagenz infolge der Bildung des durchsichtigen und beständigen Silbersols, dessen Eigenschaften vorher untersucht wurden, nach gelb und schließlich nach tiefbraun um.

Die Eichkurve zur Bestimmung des Gases in Luft nach 20stdg. Diffusion wird in Abbildung 2 wiedergegeben.

Die Lichtabsorption bei $420 \mathrm{~nm}$ steigt linear in einem weiten Konzentrationsbereich bis über $2000 \mathrm{ppm}$ (kleinste letale Konzentration). Mengen von der Größenordnung des zulässigen Grenzwertes (20-30 (ppm) können mit Sicherheit bestimmt werden.

Die an einer Probe mit 500 ppm CO erhaltenen Werte sind in Tabelle 1 aufgezeichnet.

Zur Bestimmung von $\mathrm{CO}$ im Blut wurden zwei Eichkurven an Proben von je 0,2 bzw. 0,1 ml aufgestellt;

1) p-Sulfamidbenzoesäure ist als Nebenprodukt der Saccharinherstellung leicht erhältlich. 


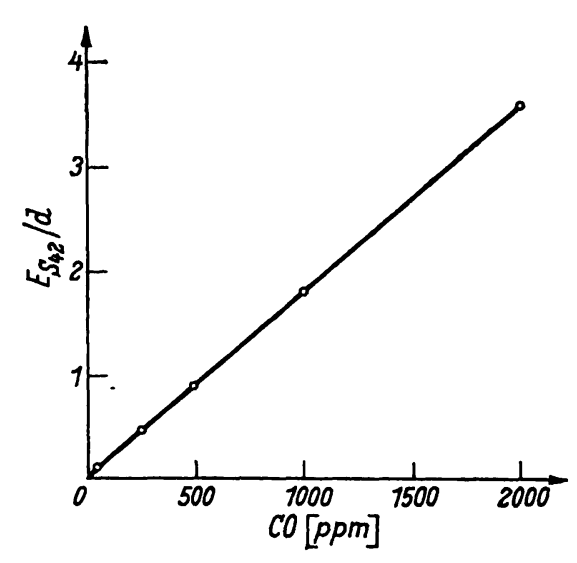

Abb. 2

Eichkurve zur CO-Bestimmung in Luft

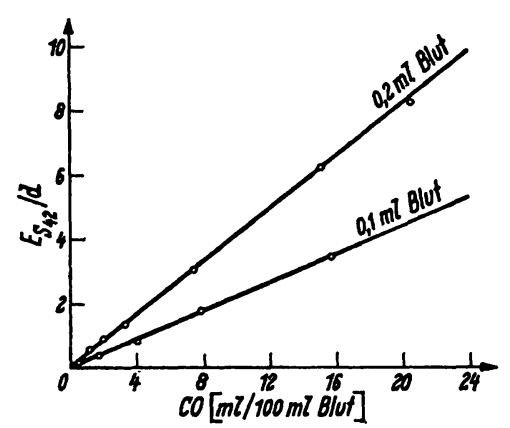

Abb. 3

Eichkurve zur CO-Bestimmung im Blut $(0,1$ bzw. $0,2 \mathrm{ml})$

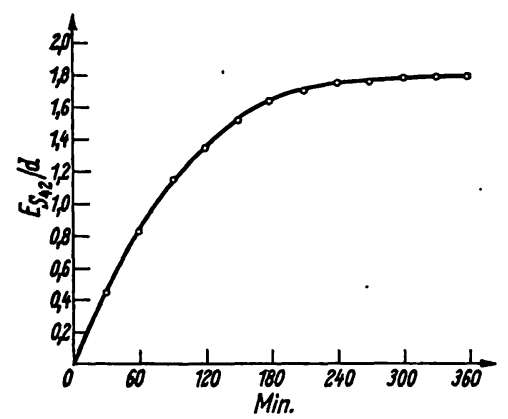

Abb. 4

Zunahme der Lichtabsorption während des Schüttelns beide nach einer Diffusionszeit von 20 Stdn. bei Normaltemperatur (Abb. 3). Wie ersichtlich, können Konzentrationen, die bei nicht exponierten Nichtrauchern auftreten $(0,2 \mathrm{Vol}-\% \mathrm{CO})$ an Blutproben von $0,1 \mathrm{~m} l$ noch bestimmt werden $\left(E_{\mathrm{S42}}: \mathrm{d}=0,04\right)$. Die Ergebnisse von Kontrollanalysen mit $0,2 \mathrm{bzw} .0,1 \mathrm{~m} l$ Blut sind in Tabelle 1 wiedergegeben.

Tab. 1

Ergebnisse der Kontrollanalysen für Luft und Blut (Wiederauffindeversuche)

\begin{tabular}{|c|c|c|c|}
\hline Vorgelegt & $\begin{array}{l}10 \mathrm{ml} \mathrm{Luft} \\
500 \mathrm{ppm} \mathrm{CO}\end{array}$ & $\begin{array}{c}0,2 \mathrm{ml} \text { Blut } \\
2,6 \text { Vol- } \% \text { Co }\end{array}$ & $\begin{array}{c}0,1 \mathrm{ml} \text { Blut } \\
1,8 \mathrm{Vol}-\% \text { Co }\end{array}$ \\
\hline $\begin{array}{r}\text { Gefunden }[\bar{x}] \\
n \\
\mathbf{s} \\
\%\end{array}$ & $\begin{array}{c}498 \mathrm{ppm} \\
10 \\
5,7 \\
1,1\end{array}$ & $\begin{array}{c}2,61 \text { Vol- } \% \\
14 \\
0,051 \\
2\end{array}$ & $\begin{array}{c}1,86 \text { Vol- } \% \text { Co } \\
10 \\
0,044 \\
2,3\end{array}$ \\
\hline
\end{tabular}

Unter den oben angegebenen Bedingungen wird $\mathrm{CO}$ auch nach $20 \mathrm{Stdn}$. nicht quantitativ absorbiert: die Lichtabsorption des Reagenz steigt weiter bei Zunahme der Kontaktdauer. Die Reproduzierbarkeit der Analysenwerte ist aber sehr befriedigend, da unter gleichen Bedingungen absorbiert wird und immer die gleiche Gasmenge reagiert.

Um die Diffusion zu fördern, wurden in einer anderen Versuchsreihe die Proben mit Reagenz und Blut mechanisch geschüttelt. An 12 Proben der gleichen Konzentration $(0,2 \mathrm{ml}$ Blut mit $3 \mathrm{Vol}-\% \mathrm{CO})$ wurde beim Photometrieren in Zeitabständen von je 30 Min. nach etwa 5 Stdn. eine konstante Lichtabsorption verzeichnet (Abb. 4).

Dementsprechend kann die Réaktion nach 5 Stdn. als quantitativ betrachtet werden. Die unter diesen Bedingungen erhaltene Eichkurve ist in Abbildung 5 ersichtlich.

Durch Schütteln wird also die Analysendauer auf 5 Stdn. abgekürzt, während die Steigung der Kurve um etwa $60 \%$ zunimmt. Beim Schütteln erfolgt auch der Um-

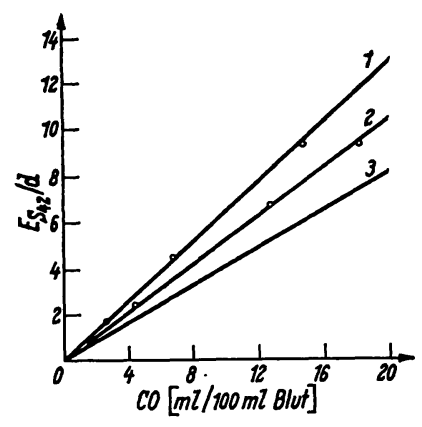

Abb. 5

Eichkurven zur Co-Bestimmung im Blut $(0,2 \mathrm{ml})$

1. 5 Stdn. Schütteln bei $20^{\circ}$

2. $20 \mathrm{Stdn}$. Stehen bei $30^{\circ}$

3. $20 \mathrm{Stdn}$. Stehen bei $20^{\circ}$

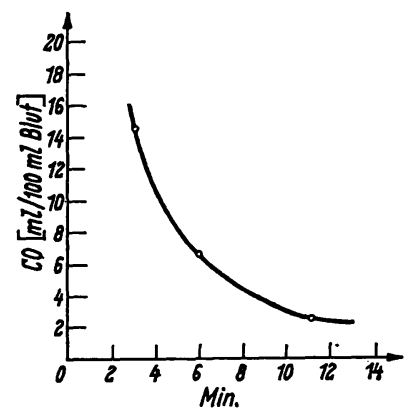

Abb. 6

Kurve zur Auswertung durch Farbumschlag

$0,2 \mathrm{~m} /$ Blut-Vergleichslösung: $\mathrm{K}_{2} \mathrm{Cr}_{2} \mathrm{O}_{7} 0,2 \mathrm{mM}$

schlag nach gelb erheblich rascher. Durch Messen der Umschlagzeit können akute CO-Vergiftungen einfach und schnell charakterisiert werden (Abb. 6).

Auch die Temperatur fördert die Diffusion (vgl. Kurven 2 und 3 in Abb. 5). Bei zwischenliegenden Temperaturen sind die erhaltenen Werte durch Interpolieren zu korrigieren.

Die angegebenen Methoden sind einfach und gut geeignet zu. Serienbestimmungen.

\section{Literatur}

1. Christmann, A. A., W. D. Block und J. Schutz, Ind. Engng. Chem., analyt. Edit. 9, 153 (1937). Chem. Ztrbl. 1937. II. 443. 2. Woods, J. T. und M. G. MeLLoN, Ind. Engng. Chem., analyt. Edit. 13, 760 (1941). - 3. Shepherd, M., Ind. Engng. Chem., analyt. Edit. 19, 77 (1947). - 4. Shepherd, M., S. Schuhman und M. V. Kilday, Analytic. Chem. 27, 380 (1955). - 5. van Slyke, D. D., A. Hiller, J. R. Weisiger und W. O. CRUz, J. biol. Chemistry 166, 121 (1946). - 6. Grékant, durch OGrer, J., Traité de Chimie 
toxicologique, Doin Paris (1924); Nrcloux, M., L'oxyde de carbone et l'intoxication oxycarbonéc, Masson Paris (1925). - 7. Kohn-Abrest, E., Précis de toxicologie, S. 37, Doin, Paris, (1955). - 8. Christmann, A. A. und E. L. Randall, J. biol. Chemistry 102, 595 (1933). - 9. Moureu, H., P. Chovin, L. Truffert und J. Lebbe, Arch. Maladies profess. Paris 2, 116 (1957). - 10. Ringold, A., P. Alto, J. R. Goldsmith, H. L. Helwig, R. FINN und F. SChUETTE, Arch. environm. Health 5, 308 (1962). - 11. Getrler, A. O. und H. C. Freimuth, Amer. J. Clin. Path. 13, 79 (1943). - 12. Seifert, P. und L. Schmieder, Dtsch. Zschr. gerichtl. Med. 41, 435 (1952). - 13. SACHs, V., DrägerHefte 235, 542 (1959). - 14. Gramer, L., Wschr. Unfallhk. 4, 121 (1961); Zbl. Arbeitsmed. Darmstadt 3, 53 (1961); Ärztl. Laborat. 12, 373 (1961). - 15. Massmann, W., Zschr. inn. Medizin 7, 293 (1956). - 16. Rovghton, F. J. W. und P. F. Scholander, J. biol. Chemistry 148, 551 (1943). - 17. Berninger, H. und R. Smith, Clin. Chem. N. Y. 5, 127 (1959). - 18. Franzen, E. und H. ThIEde, Z. ges. Hygiene Grenzgeb. 1, 44 (1964). - 19. FrETwURST, F. und F. Meinecke, Arch. Toxikol. 17, 273 (1959). - 20. Giubileo, M., Lavoro e med., Genova 49, 530 (1958). - 21. KuRz, H. und H. Dirkwaller, Arch. Toxikol. 15, 291 (1955). - 22. Zrjlstra, T. M., Klin. Wschr. 34, 384 (1956). - 23. WolfF, E., Nord. kriminaltekn. Tidskr. 11, 125 (1941); Ann. méd. lég. Paris 27, 221 (1947). - 24. Wennesland, R., Acta physiol. Scand. 1, 49 (1940). - 25. Allen, T. H. und W. S. Roor, J. biol. Chemistry 216, 319 (1955). - 26. Berka, J., Acta med. Scand. 152, 485 (1955). - 26 a. Fabre, R., R. Truhaut und F. Berrod, Ann. pharm. franr.. 9, 626 (1951). - 27. Feldstern, M. und N. C. KLendshoj, Chem. Abstr. 49, 410 b (1955). - 28. Le MOAN, G., Ann. pharmaceut, franç. 10,
269 (1952). - 29. Marquardr, W., Dtsch. Zschr. gerichtl. Med. 40, 385 (1951). - 30. MassmanN, W. und D. Sprecher, Arch. Gewerbepath. Berlin 14, 208 (1956). - 31. Nestorescu, B. und A. Roventa, Igiena 2, 165 (1959). - 32. Nitschke, M., Ann. méd. lég. Paris 33, 155 (1953). - 33. Truhaut, R. und C. Boudéne, Compt. Rend. Acad. Sci. France 256, 5453 (1963). - 34. Werst, H. J., Z. Ges. Hygiene 7, 504 (1961). - 35. Williams, L. A., R. A. LINN und B. ZaK, Amer. J. Clin. Path. 34, 334 (1960). - 36. Cruhandu, G. und V. Rusu, Z. analyt. Chem. 222, 393 (1966). - 37. Jones, J. G. und D. H. Walters, Ann. Occup. Hygiene 5, 221 (1962). - 38. Vignoli, L., Bl. Cristau, J.-P. Defretin und R. Vignoli, Arch. Mal. profess. Paris 21, 423 (1960). - 39. Ciunandu, G., Studii Cercetări Chimie 3/4, 189 (1956). - 40. FAcsko, G. und R. Minges, Talanta (London) 5, 102 (1960). - 41. Ciuhandu, G., D. Ceaușescu und G. Facsko, Studii Cercetări Chimie 1, 79 (1957). - 42. Cruhandu, G. und V. Gruran, Z. analyt. Chem. 159, 250 (1958). - 43. Crumandu, G., Studii Cercetări Chimie 3/4, 243 (1955). - 44. Ciuhandu, G., G. Krall und V. Giuran, Acta chim. Acad. Sci. hung. 28, 171 (1961). - 45. Ciuhandu, G. und G. Krall, Z. analyt. Chem. 172, 81 (1960). - 46. Ciuhandu, G., Studii Cercetări științ. 1/4, 133 (1955). - 47. Cruhandu, G., Z. analyt. Chem. 155, 321 (1957); 161, 345 (1958). - 48. Ciunandu: G. und G. KraII, Rev. Chimie 9, 531 (1959). - 49. Ciuhandu, G., V. Rusu und M. Diaconovicr, Z. analyt. Chem. 208, 81 (1965). 50. Levaggr, D. A. und M. Feldsten, Amer. ind. Hyg. Assoc. Quart. 25, 64 (1964). - 51. Cruhandu, G. und V. Rusu, Proceedings XV. Internat. Congr. Occup. Health, Wien 1966, S. 307. 52. Feldstein, M., J. forensic. Sci. 10, 1, 35 (1965).

Dr. G. Ciuhandu Str. Michelangelo 1 Timișoara, Rumänien

\title{
Dünnschichtchromatographische Trennung und halb-quantitative Bestimmung des Blut-Phenylalanins als Verfahren zur systematischen Prüfung auf Phenylketonurie bei Kleinstkindern
}

\author{
Von E. NITSChKE \\ Aus dem Institut d'Hygiène et de Santé Publique, Luxemburg (Direktor: Dr. F. Scbwacbtgen)
}

(Eingegangen am 4. Oktober 1967)

Es wird eine dünnschichtchromatographische Methode zur scharfen Trennung und zur halb-quantitativen Bestimmung des Plasmaphenylalanins angegeben. Der Phenylalanin-Fleck befindet sich, klar getrennt, über denjenigen der anderen Aminosäuren. Zur Verwendung gelangen Merck Fertigplatten Silicagel $\mathrm{F}_{254}$. Eine neue, spezifische Fluoreszenzmethode gestattet die einwandfreie Bestätigung der Befunde, so daß falsch-positive Resultate ausgeschlossen sind.

Wegen ihrer sicheren Aussage, ihrer Einfachheit und Billigkeit wird sie in unserem Land als screening test für die eben anlaufende systematische Untersuchung auf Phenylketonurie bei Kleinstkindern benutzt.

A thin layer chromatographic method is reported for the clean separation and semi-quantitative determination of phenylalanine in blood plasma. Ready-prepared plates of silicagel $\mathrm{F}_{254}$ (Merck) were used. The phenylalanine spot is clearly separated from all the other amino acids, and its identity is confirmed by a new, specific fluorescence reaction, which excludes any false positive results.

Owing to its specificity, simplicity and cheapness, we now use this method in the routine phenylketonuria screening test on newly born infants, which has just started in this country.

Ursache der Phenylketonurie ist die Abwesenheit eines spezifischen Enzyms, der Phenylalanin-hydroxylase ${ }^{1}$ ) im Organismus, wodurch die normale Umwandlung des Phenylalanins in Tyrosin nicht stattfinden kann. Es ent-

1) Enzyrne: Phenylalaninhydroxylase = Phenylalanin-4-hydroxylase (EC 1.99.1.2); L-Aminosäureoxydase = L-Aminosäure: $\mathrm{O}_{2}$ Oxydoreduktase (desaminierend) (EC 1.4.3.2). steht eine starke Anbäufung von Phenylalanin bis auf das 10-20fache der Normalwerte und gleichzeitig über einen Stoffwechselseitenweg eine starke Vermehrung der Phenylbrenztraubensäure, der Phenylmilchsäure, der Phenylessigsäure u. a. $\mathrm{m}$. Die Phenylbrenztraubensäure kann sehr leicht im Harn durch Eisenchlorid nachgewiesen werden (Phenistix-Teststäbchen). Diese Re- 